\title{
Abnormal interneuron development in disrupted-in-schizophrenia-1 L100P mutant mice
}

\author{
Frankie HF Lee ${ }^{1,2}$, Clement C Zai ${ }^{1}$, Sabine P Cordes ${ }^{3,4}$, John C Roder ${ }^{3,4}$ and Albert HC Wong 1,25,6*
}

\begin{abstract}
Background: Interneuron deficits are one of the most consistent findings in post-mortem studies of schizophrenia patients and are likely important in the cognitive deficits associated with schizophrenia. Disrupted-in-Schizophrenia 1 (DISC1), a strong susceptibility gene for schizophrenia and other mental illnesses, is involved in neurodevelopment, including that of interneurons. However, the mechanism by which DISC1 regulates interneuron development remains unknown. In this study, we analyzed interneuron histology in the DisC1-L100P single point mutation mouse, that was previously shown to have behavioral abnormalities and cortical developmental defects related to schizophrenia.

Results: We sought to determine whether a Disc1-L100P point mutation in the mouse would alter interneuron density and location. First, we examined interneuron position in the developing mouse cortex during embryonic days 14-16 as an indicator of interneuron tangential migration, and found striking migration deficits in DisC1-L100P mutants. Further analysis of adult brains revealed that the Disc1-L100P mutants have selective alterations of calbindin- and parvalbumin-expressing interneurons in the cortex and hippocampus, decreased GAD67/PV co-localization and mis-positioned interneurons across the neocortex when compared to wild-type littermates.

Conclusion: Our results are consistent with the anomalies seen in post-mortem schizophrenia studies and other Disc1 mutant mouse models. Future research is required to determine the specific mechanisms underlying these cellular deficits. Overall, these findings provide further evidence that DISC1 participates in interneuron development and add to our understanding of how DISC1 variants can affect susceptibility to psychiatric illness.
\end{abstract}

Keywords: Disrupted-in-Schizophrenia 1 (DISC1), Interneuron, Mutant mouse, Schizophrenia

\section{Background}

Cognitive control depends on neural synchrony that maintains a balanced excitation and inhibition in different brain regions [1]. GABAergic interneurons are critical for providing inhibitory control over pyramidal neurons and modulating synchronized oscillations [2]. Interneuron deficits have been one of the most consistent findings in human post-mortem schizophrenia studies, including reductions in glutamic acid decarboxylase-67 (GAD67) expression, and parvalbumin (PV) mRNA expression and immunoreactivity [3-5]. Different interneuron subtypes have distinct electrophysiological and synaptic

\footnotetext{
*Correspondence: albert.wong@utoronto.ca

'Campbell Family Mental Health Research Institute, Centre for Addiction and Mental Health, 250 College Street, Toronto, ON M5T 1R8, Canada

${ }^{2}$ Department of Pharmacology, University of Toronto, Toronto, ON M5S 1A8, Canada

Full list of author information is available at the end of the article
}

characteristics [6]. In schizophrenia, GAD67 reduction appears to be restricted to PV-interneurons $[7,8]$. This is of particular relevance as recent optogenetic studies on animal models have shown that PV-interneurons are required for generating gamma-frequency oscillations $[9,10]$, that are critical for cognition [11,12]. Consistent with this notion, schizophrenia patients display abnormal neural oscillations and synchronizations [13,14]. Furthermore, rodents with loss of PV-interneurons and impaired gamma activity show selective cognitive deficits reminiscent of schizophrenia symptoms $[15,16]$.

Disrupted-in-schizophrenia 1 (DISC1) is a strong susceptibility gene for schizophrenia and other mental disorders [17]. DISC1 functions as a scaffold protein and regulates a wide-range of neurodevelopmental processes [18-20]. Different mutant DISC1 mouse models have displayed selective reductions in PV interneurons [21-24] and alterations in their laminar distribution [22].

\section{Biomed Central}


Recently, Steinecke et al. demonstrated that DISC1 also regulates interneuron tangential migration [25], further supporting a possible role for DISC1 in modulating interneuron development.

Our group previously described a mutant mouse line, Disc1-L100P that has behavioral and cognitive abnormalities related to schizophrenia [26], consistent with four other publications [27]. Given the accumulating evidence for DISC1 and interneuron abnormalities in schizophrenia, we undertook a comprehensive histological analysis of interneurons in the Disc1-L100P mutants. Our findings suggest that Disc1 mutations may have distinct spatial and temporal effects in different interneuron subtypes. Overall, our study provides evidence for the effects of Disc1 SNPs on interneuron development that represent a starting point for further investigations into developmental and pathophysiological mechanisms in schizophrenia.

\section{Results}

Impaired tangential interneuron position in the embryonic Disc1-L100P mouse

Recent evidence suggests that DISC1 is likely to play an important role in interneuron tangential migration $[25,28]$. Thus, we compared the tangential migratory pathways in embryonic mouse brains of wild-type (WT) and Disc1-L100P mutants at two different time points, E14 and E16 by immunostaining with an early interneuron marker, calbindin (CB) [29] (Figure 1A). At E14, Disc1L100P mutants had a lower proportion of CB-cells in bin $6(23.73 \pm 1.46 \%)$ while slightly higher in bin 7 when compared to WT (bin 6: $28.47 \pm 1.02 \%$ ) (Figure 1B). But at E16, we observed significantly more CB-interneurons in the dorsal cortex of bins 3 and 4 in WT controls (bin 3: $4.44 \pm 0.7 \%$; bin $4: 12.56 \pm 1.62 \%)$ when compared to the Disc1-L100P mutants (bin 3: 2.14 $\pm 0.24 \%$; bin 4: $7.62 \pm$ $1.69 \%)$, while a larger proportion remained near the ventral sites of bin 6 in the mutant mice (WT: $27.24 \pm 1.75 \%$; L100P: $36.14 \pm 2.3 \%$ ) (Figure 1C). This indicates that migrating interneurons in the Disc1-L100P mutants failed to reach their proper dorsal target position, suggesting that the L100P mutation in the Disc1 gene is likely to disrupt the tangential migration of interneurons.

\section{Altered CB- and PV-expressing interneuron numbers in the MPFC and DLFC of Disc1-L100P mutant mice}

To address whether interneuron deficits remain as pronounced in our Disc1-L100P mouse model or become more diffuse as seen in post-mortem analyses, we examined the number of interneurons in both the medial prefrontal cortex (mPFC) and dorsolateral frontal cortex (DLFC) of the adult mouse (Figure 2A). Previous mutant DISC1 animal studies have demonstrated that interneuron deficits are present in these mouse brain regions [21-24]. Two different subclasses of interneurons (CB and PV) were immunolabeled with their respective antibodies, and labeled cells were counted in WT and Disc1-L100P mutants. In the mPFC, we observed significantly fewer PV-interneurons in L100P mutants $(152.48 \pm 7.46)$ when compared to WT $(199.27 \pm$ 7.28), consistent with previous reports [22]. However, there was no significant difference in $\mathrm{CB}$-interneuron density (Figure 2B). Interestingly, L100P mutants had more CB-labeled cells $(1008.31 \pm 44.29)$ within the DLFC compared to WT $(862.15 \pm 43.06)$, while no alterations were observed with PV-labeled cells (Figure 2C). In addition, the average overall number of both interneuron subtypes was significantly higher in WT controls than the mutants within the mPFC, but there was no difference within the DLFC (Additional file 1: Figure S1). Our results show that Disc1 mutations affect the numbers of each interneuron subtype differently.

\section{Aberrant interneuron laminar position in Disc1-L100P mutants}

Post-mortem human and mutant DISC1 animal studies have reported abnormally located interneurons within cortical layers $[8,22,30]$. In our study, we investigated interneuron laminar position by analyzing CB- and PVimmunolabeled cells in a series of equally-spaced regions spanning the thickness of the cerebral cortex within the DLFC. We found an abnormal distribution of both interneuron subtypes in Disc1-L100P mice. The percentage of CB-interneurons was significantly higher in octants 3 and 4 of Disc1-L100P mutants (octant 3: $13.98 \pm$ 0.91\%; octant 4: 7.94 $\pm 0.43 \%$ ) compared to WT (octant 3: $9.58 \pm 0.58 \%$; octant 4: $5.52 \pm 0.34 \%$ ), while WT controls exhibit a more dispersed pattern with higher percentages located in superficial and deeper cortical layers (Figure 3A). Conversely, the distribution pattern of PVinterneurons was similar between WT and Disc1-L100P mutants, but mutants displayed a shift towards more superficial cortical layers with a significantly higher proportion in octant $1(6.32 \pm 0.5 \%)$ and lower in octant 6 $(13.68 \pm 0.42 \%)$ versus WT controls (octant 1: $3.81 \pm$ 0.22\%; octant 6: $15.28 \pm 0.47 \%$ ) (Figure 3B). These data suggest an aberrant localization of cortical interneurons in Disc1-L100P mice that varies with each interneuron subtype.

\section{More PV-interneurons located in the lateral neocortex of Disc1-L100P mice}

Interneurons migrate from the ganglionic eminence of the telencephalon, moving from the lateral to medial cortex [31]. To further support our previous results of tangential migration deficits, we examined the distribution of interneurons across the medial-lateral axis of the neocortex in adult WT and Disc1-L100P mutant mice 

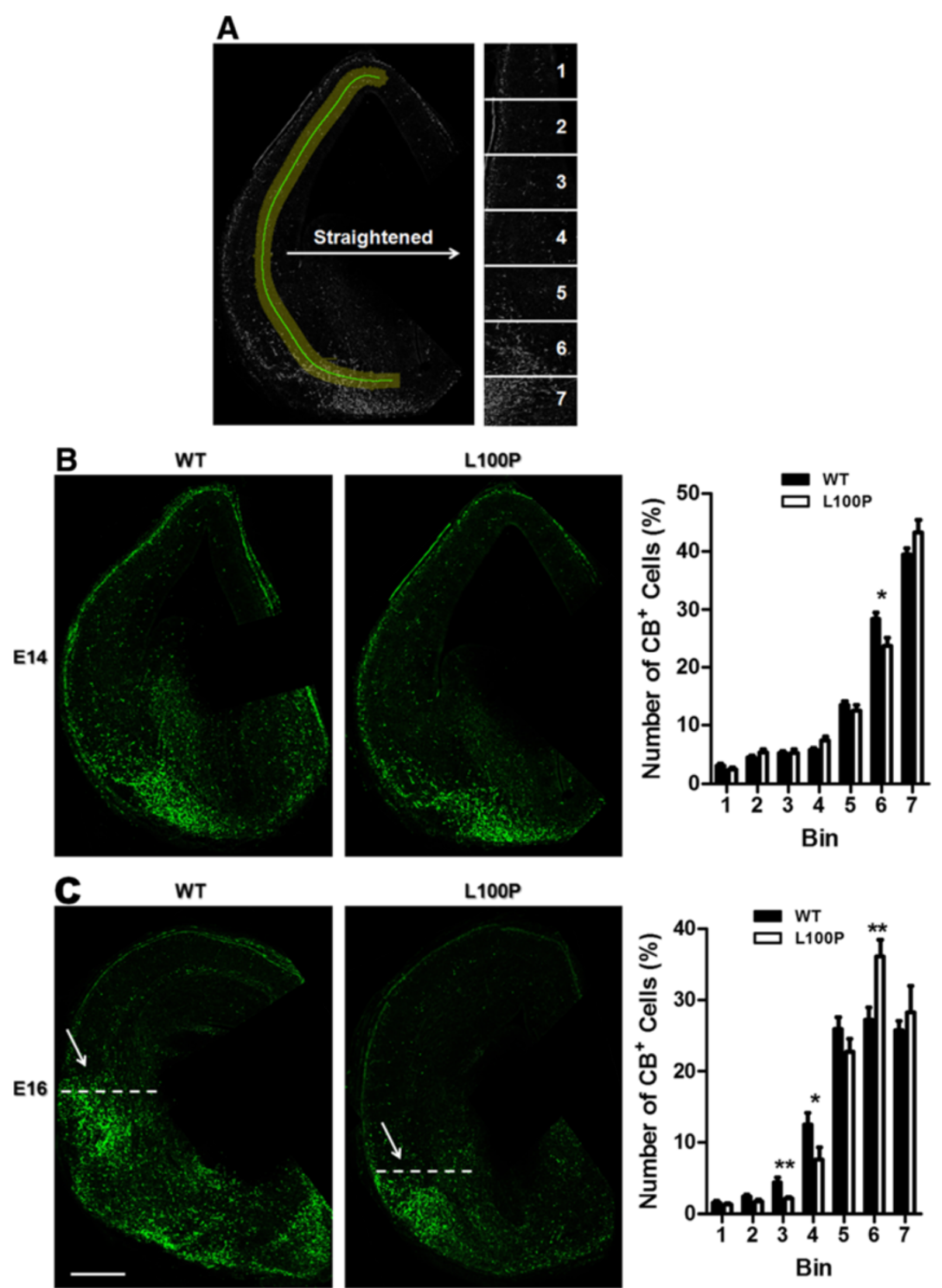

Figure 1 Disrupted interneuron tangential migration in Disc1-L100P embryonic mice. (A) Coronal sections of either E14 or E16 embryonic brains immunostained with CB were straightened and divided into seven equidistant bins covering the tangential migratory pathway of interneurons. Fluorescently-labeled cell numbers were counted in each bin and normalized to the total number in all bins. The distribution of these interneurons was significantly different for both E14 and E16 time points between WT and Disc1-L100P (two-way ANOVA, $p<0.01$ ). (B) E14 CB-immunolabeled images of WT and Disc1-L100P mice are shown on the left. Disc1-L100P mice had a significantly lower proportion of CB-cells in the ventral bin 6 when compared to WT controls $(n=25-28$ sections from 4 different embryos per group, $t$-test, $p<0.05)$. (C) At E16, a more pronounced difference in interneuron tangential migration was observed between WT and DisC1-L100P mice (white arrows). Disc1-L100P mutants had significantly less $\mathrm{CB}^{+}$cells in bins 3 and 4, while more remained near the ventral bin 6 when compared to WT ( $n=19-24$ sections from 4 different embryos per group, $t$-test, $p<0.01$ ). Scale Bar, $300 \mu \mathrm{m}$. All data are shown as mean $\pm \mathrm{SEM}^{*}{ }^{*} p<0.05,{ }^{* *} p<0.01$ versus WT. CB, calbindin.

(Figure 4A). We found no substantial change in the distribution of $\mathrm{CB}$-interneurons across the neocortex (Figure 4B). However for PV-interneurons in Disc1L100P mutants, a higher proportion of fluorescent cells were situated in the lateral cortex and fewer reached the medial region when compared to WT controls (two-way ANOVA, Bin 1: WT $-18.67 \pm 1.98 \%$, L100P $-23.52 \pm$ $1.02 \%$; Bin 4 : WT $-26.34 \pm 1.51 \%$, L100P $-23.7 \pm$ 
$0.62 \%$ ) (Figure 4C). These results indicate that the Disc1-L100P mutation only affected the tangential positioning of PV interneurons within the cortex, which may reflect a defect in interneuron tangential migration.

\section{Less GAD67/PV co-localization in Disc1-L100P mice}

One of the most robust findings in post-mortem schizophrenia studies is a reduction in GAD67 expression, preferentially within PV-interneurons [7,8]. However, there is still a lack of evidence for the effect of DISC1 on GAD67 expression. Hence we sought to determine the co-localization of GAD67 and PV in our Disc1-L100P mutants (Figure 5A). Consistent with previous reports, we found significantly fewer $\mathrm{GAD} 67^{+} \mathrm{PV}^{+}$cells in the Disc1-L100P mutants $(93.57 \pm 0.81 \%)$ vs. WT (97.93 \pm $0.40 \%$ ) (Figure $5 \mathrm{~B}$ ), indicating that DISC1 may affect GAD67 expression.

\section{Increased hippocampal PV-interneurons in Disc1- L100P mutants}

There is evidence for hippocampal interneuron deficits in schizophrenia [32]. Thus, we measured both CB- and PVinterneuron density within all subfields of the hippocampus in Disc1-L100P mice (Figure 6A). Surprisingly, we observed significantly more $\mathrm{PV}^{+}$cells within the hippocampus specifically in the CA 1 and CA2/3 regions of the Disc1-L100P mutants (CA1: 12.64 \pm 1.36 ; CA2/3: $23.45 \pm$ 1.73) when compared to WT controls (CA1: $7.92 \pm 0.87$; CA2/3: $14.67 \pm 1.1$ ) (Figure 6B). CB-labeled cell numbers were not significantly different in all hippocampal subfields (Figure 6C). Our data are inconsistent with previous reports of fewer hippocampal PV-interneuron numbers in schizophrenia, but suggest that the Disc1-L100P mutation results in more $\mathrm{PV}^{+}$cells.

\section{Discussion}

There is substantial evidence for an association between DISC1 and several major mental illnesses. However, the mechanism by which DISC1 gene variants produce both cellular and behavioral abnormalities is still unclear. In this study, we examined embryonic interneuron tangential migratory position and adult histology of two interneuron subtypes (CB and PV) in a mouse with a point mutation in the Disc1 gene (L100P), which has been previously shown to have behaviors relevant to schizophrenia [26].

A recent study suggests that DISC1 is necessary for proper tangential migration of cortical interneurons [25]. Therefore, we examined the tangential migratory pathway of interneurons at E14 and E16, as an indicator of migration. Consistent with the putative role for DISC1 in interneuron development, our study revealed that the Disc1-L100P mutants displayed abnormal tangential migration. This was further supported by our findings that
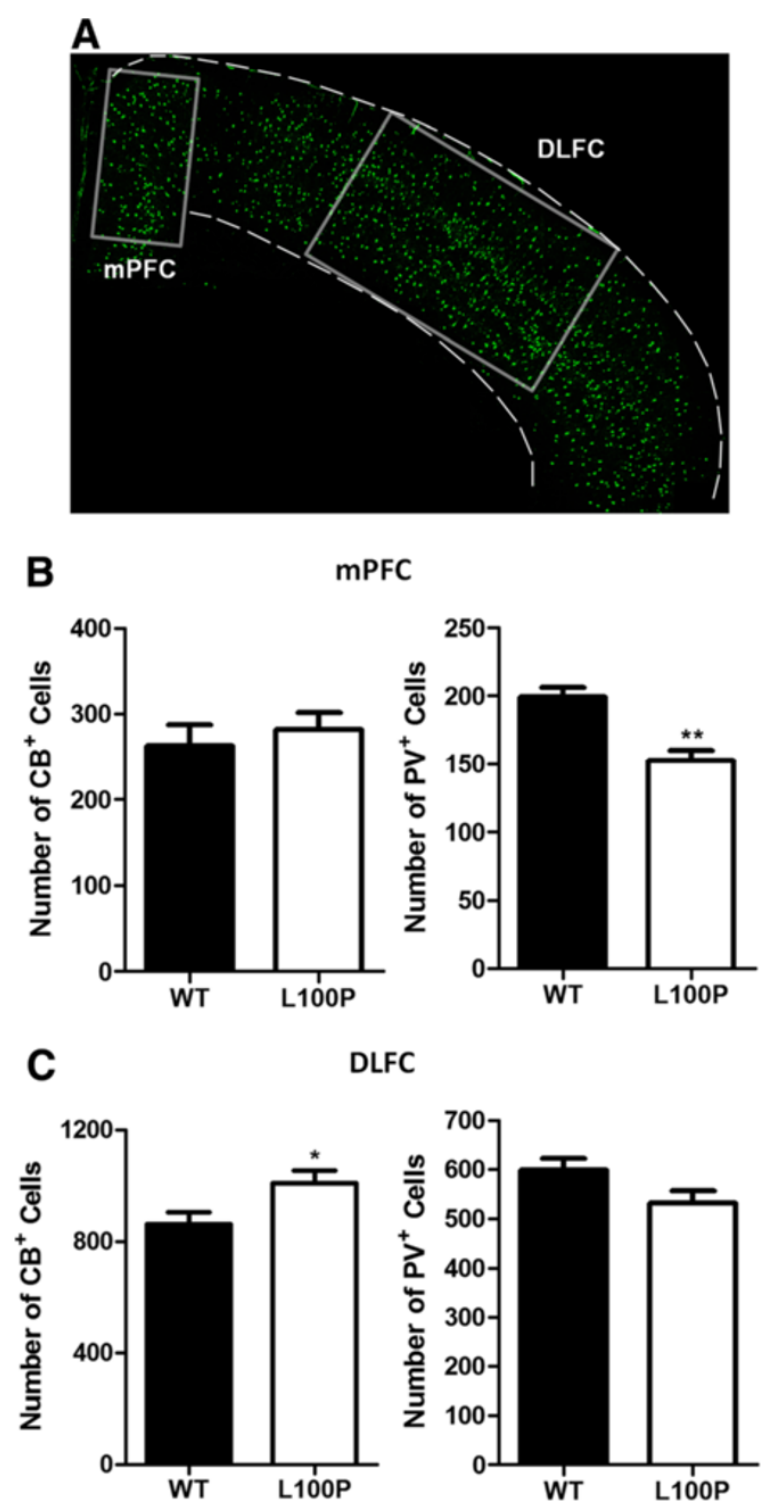

Figure 2 Differential alterations in CB- and PV-labeled interneurons in the MPFC and DLFC of Disc1-L100P adult mice. (A) A representative section used for counting CB- and PV-labeled cell densities is shown with boxes depicting the areas analyzed for the $\mathrm{mPFC}$ and DLFC regions. Scale Bar, $500 \mu \mathrm{m}$. (B) Quantification of $\mathrm{CB}^{+}$and $\mathrm{PV}^{+}$cells in the mPFC showed significantly fewer PV-interneurons in the Disc1-L100P mutants but no significant difference with CB-interneurons when compared to WT $(n=13-14$ from 4 mice per group; $t$-test, $p<0.01$ ). (C) In contrast, Disc1-L100P mutants have significantly more $\mathrm{CB}^{+}$cells but not $\mathrm{PV}^{+}$cells in the DLFC versus WT ( $n=21-22$ from 4 mice per group, $t$-test, $p<0.05$ ). All data are shown as mean \pm SEM; ${ }^{*} p<0.05$, ** $p<0.01$ versus WT. $C B$, calbindin; DLFC, dorsal lateral frontal cortex; $\mathrm{mPFC}$, medial prefrontal cortex; PV, parvalbumin.

PV-interneurons remained in the lateral adult cortex and that there were fewer interneurons overall in the mPFC. A plausible explanation is that the L100P mutation disrupts specific DISC1 protein interactions and results in mis- 
regulated downstream signals. ErbB4, and its substrate Neuregulin-1 (NRG1), have been extensively studied for their role in interneuron tangential migration [33-35]. DISC1 has been hypothesized to converge with NRG1ErbB4 cascades in modulating migration [28]. However, interneuron tangential migration deficits are likely to arise through the simultaneous dysregulation of not just one, but several protein interactions including cytoskeletal proteins [31], dysbindin [2], neurotrophins [36] and transcription factors [37-39]. Future research addressing how DISC1 can affect these various pathways will help to elucidate the precise molecular mechanisms by which DISC1 affects interneuron tangential migration.

Next we examined the number and positioning of interneurons in adult Disc1-L100P mice and found changes consistent with human post-mortem schizophrenia studies including reductions in PV immunoreactivity and abnormal laminar distribution patterns [4,8,30]. Interestingly, other mutant Disc1 mouse models exhibit similar reductions of PV-interneurons in the PFC and aberrant cortical positioning [21-23]. This suggests that DISC1 protein disruptions may overlap among these different mouse models, with a common effect on interneuron genesis and incorporation of PV-interneurons into proper cortical layers. Interneuron genesis in the ganglionic eminence is likely to be controlled by different transcription factors [40], but the relationship between DISC1 and interneuron production remains to be determined.

Another theory is based on the pyramidal interneuron network gamma (PING) model, which suggests that PVinterneurons are recruited by glutamatergic inputs from pyramidal neurons [41]. Previously, misplaced cortical pyramidal neurons and reduced spine densities within layers III and V pyramidal neurons were found in the Disc1L100P mutants [42]. Consequently, incorrect guidance cues and weakened excitatory drive may lead to less recruitment of PV-interneurons and aberrant cortical lamination [43].

Interestingly, the increase in $\mathrm{CB}$ immunoreactivity within the DLFC and PV-immunoreactivity within the CA1 and CA2/3 subfields of the hippocampus did not parallel those observed in post-mortem schizophrenia studies [44-46] and
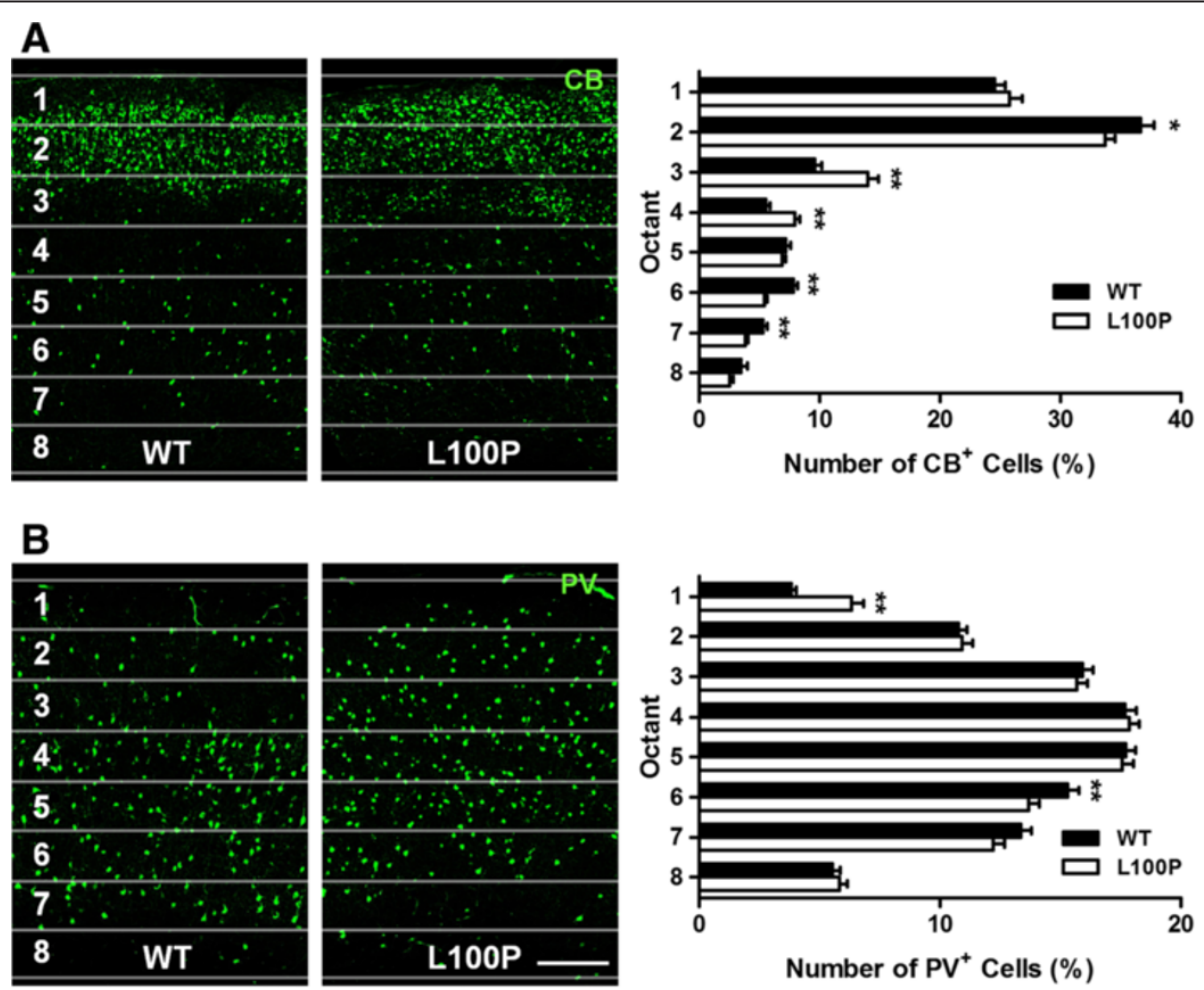

Figure 3 Altered cortical interneuron laminar distribution in Disc1-L100P adult mice. Quantification of both CB- and PV-labeled cells was achieved by counting the number of fluorescent cells in each octant and expressed as a percentage of total number per ROI of fixed size across the neocortex. (A) There was a significant interaction effect with CB-interneurons across the octants between WT and DisC1-L100P mice (two-way ANOVA, $p<0.01$ ) with proportionally more CB-interneurons in the middle cortical layers of octants 3 and $4(t$-test, $p<0.01$ ). Conversely, there were fewer $\mathrm{CB}^{+}$cells in the second, sixth and seventh octants in the Disc1-L100P mutant versus WT ( $n=52$ ROls from 4 different mice per group; $t$-test, $p<0.01$ ). (B) PV-labeled interneurons were significantly different in their distribution across the octants between WT and Disc1-L100P mutants (two-way ANOVA, $p<0.01$ ). More PV + cells were observed in WT of octant 6 but less in superficial cortical layers of octant 1 than in the mutants ( $n=84-90$ ROls from 5 different mice per group; $t$-test, $p<0.01$ ). Scale Bar, $300 \mu$ m. All data are shown as mean \pm SEM; ${ }^{*} p<0.05$, ** $p<0.01$ versus WT. CB, calbindin; PV, parvalbumin; ROI, region of interest. 


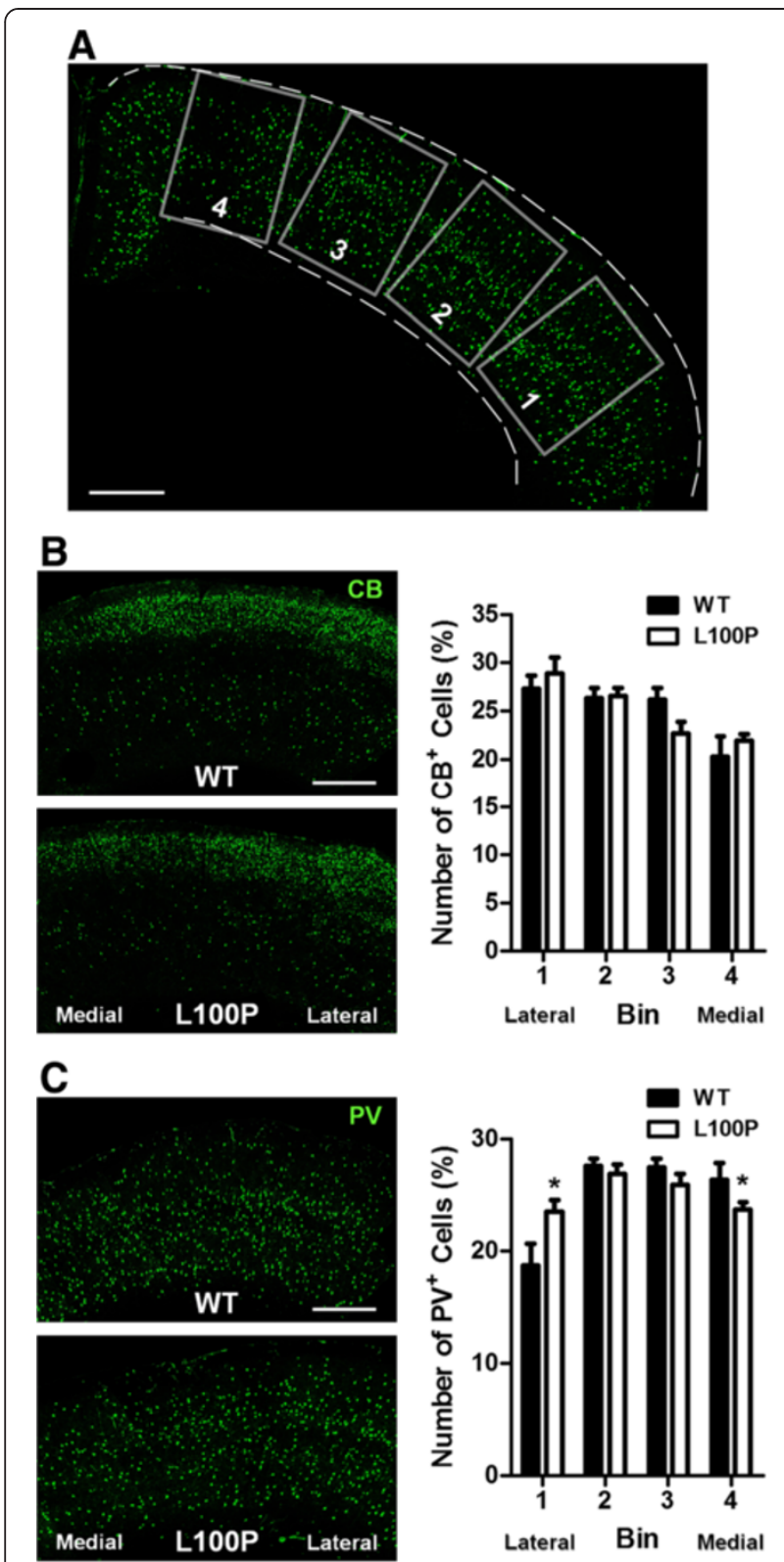

Figure 4 Aberrant tangential distribution of PV-interneurons in Disc1-L100P adult mice. (A) Four sampling ROls immunolabeled with either $\mathrm{CB}$ or $\mathrm{PV}$ antibodies were outlined across the neocortex along the medial-lateral axis. The number of $\mathrm{CB}^{+}$and $\mathrm{PV}^{+}$cells were counted and expressed as a percentage of total cells in each ROI. (B) There was no significant difference in the tangential distribution of $\mathrm{CB}^{+}$cells between WT and DisC1-L100P mice ( $n=12-13$ ROls from 4 different mice per group). (C) PV-labeled cells were distributed differently depending on genotype. The Disc1-L100P mutants had a significantly higher proportion of $\mathrm{PV}^{+}$cells located laterally (bin 1) but fewer $\mathrm{PV}^{+}$cells in medial bin 4 when compared to $\mathrm{WT}(\mathrm{n}=22$ ROls from 4 different mice per group; two-way ANOVA, $t$-test, $p<0.05)$. Scale Bar, $500 \mu \mathrm{m}$. All data are shown as mean \pm SEM; * $p<0.05$ versus WT. CB, calbindin; PV, parvalbumin; ROI, region of interest. a truncated Disc1 mouse model [22]. Despite the inconsistent findings in the literature, an increase in CB mRNA expression and immunoreactivity in the PFC has been reported in several post-mortem studies [47,48]. Compared to PV subpopulations, CB-interneurons are less extensively studied and thus their features in schizophrenia remain unclear. CB-interneurons may affect pyramidal neuron activity in a different way than PV-interneurons, since the two interneuron types have different electrophysiological and synaptic characteristics [6]. Furthermore, the increase in CB-interneurons may be a compensatory response to PVinterneuron reductions [47]. Moreover, DISC1 can have differential regional effects between the cortex and hippocampus, as evident from opposing neuronal migration and outgrowth effects in previous DISC1 knockdown studies $[49,50]$. Multiple pathways are likely to be involved in determining interneuron fate. Further research is required to elucidate the precise relationship between DISC1-related pathways and to understand the specific roles of DISC1 in different interneuron subpopulations.

As mentioned previously, reduced GAD67 expression in PV-expressing cells has been consistently reported in post-mortem brains of schizophrenia patients $[7,8]$. Here, we provide novel evidence of diminished GAD67/PV colocalization in Disc1-L100P mutants when compared to WT controls. Immunohistochemical analyses have confirmed the co-expression of DISC1 and GAD67 in GABAergic interneurons [51]. The Disc1 L100P mutation may affect specific downstream transcription control of GAD67 enzyme levels, or GAD67 reduction may be a compensatory response to reduced PV immunoreactivity. Furthermore, western blots of GAD67 can provide information on whether GAD67 protein levels are changed in our Disc1 mutants. The causes and functional relationship between DISC1 and GAD67 remain to be determined. Our findings provide a starting point for future research to elucidate the role of DISC1 in GABAergic signaling.

As mentioned previously, the immunoreactivity and distribution patterns of PV-immunostained cells have been extensively studied in human post-mortem and animal studies. However, the histological relationship between different interneuron subpopulations has not been examined. Given that our Disc1-L100P mutants displayed selective alterations in density and distribution of both PVand CB-immunostained cells, future double interneuron immunolabeling experiments would provide important insights about whether the density and distribution of one interneuron subtype is associated with the other.

In conclusion, the results presented in this study support the notion that DISC1 plays a role in interneuron development. But whether DISC1 mutations are a primary cause of aberrant interneuron development through direct disruption of interactions with relevant proteins and transcription factors or produce secondary effects from 

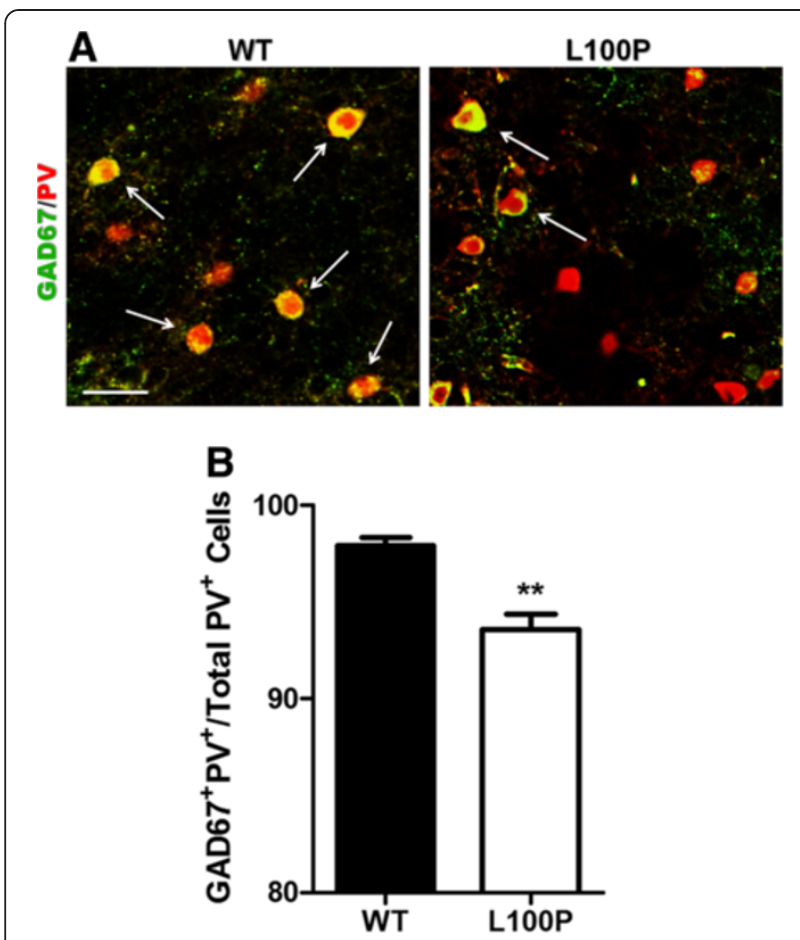

Figure 5 Reduced GAD67/PV co-localization in Disc1-L100P adult mutants. (A) GAD67 (green) and PV (red) fluorescently labeled images in WT and Disc1-L100P mutants. White arrows indicate those with more than 50\% overlap of GAD67 and PV. Scale Bar, $50 \mu \mathrm{m}$. (B) Quantification of the percentage of $\mathrm{GAD} 67^{+} \mathrm{PV}^{+}$cells per total $\mathrm{PV}^{+}$ cells revealed significantly less GAD67 expression within PV-cells in Disc1-L100P mice compared to WT ( $n=106-128$ ROls from 4 different mice per group). All data are shown as mean \pm SEM; ${ }^{* *} p<0.01$ versus WT. GAD67, glutamic acid decarboxylase (67 kDa); PV, parvalbumin.

disturbed pyramidal neuron positioning, remains to be determined. Moreover, investigating electrophysiological properties of the Disc1-L100P mouse cortex and the hippocampus [52] would be useful in addressing functional outcomes of these histological abnormalities. Nonetheless, we have provided an overview of interneuron histology and development in an $N$-ethyl- $N$-nitrosourea (ENU)-induced Disc1-L100P mouse line, which supplements our previous work in characterizing cortical abnormalities of pyramidal neurons [42]. Our findings further support the role of DISC1 in interneuron development and provide additional insights about how Disc1 mutations can lead to the brain and cognitive abnormalities associated with schizophrenia. More importantly, this study represents a starting point for further investigation of DISC1-related mechanistic pathways in interneuron development.

\section{Methods}

Mice

$N$-ethyl-N-nitrosourea (ENU)-mutagenized Disc1-L100P homozygous mutant embryonic and adult mice (8 weeks) on a C57BL/6 background were generated as previously described [26]. WT littermates from the same breeding batch were used as controls. All mouse protocols were approved by the Centre for Addiction and Mental Health Animal Care Committee.

\section{Immunohistochemistry}

Adult mice were sacrificed by cervical dislocation. Both embryonic and adult mouse brains were dissected, fixed in $4 \%$ paraformaldehyde, cryoprotected in 30\% sucrose and stored at $-80^{\circ} \mathrm{C}$ before further processing. Frozen coronal sections of $10 \mu \mathrm{m}$-thickness were cut using a cryostat (Bright Instrument Co. 5030). All sections were initially incubated in blocking solution (0.1M PBS, $1 \%$ Triton X100, $0.5 \%$ Tween 20, 5\% skim milk) for 2 hours at room temperature to reduce nonspecific background, followed by primary antibodies overnight at $4^{\circ} \mathrm{C}$ and secondary antibodies for 2 hours at room temperature. The following primary antibodies were used: anti-parvalbumin (1:200; Sigma-Aldrich), anti-calbindin D-28k (1:200; Millipore) and anti-GAD67 (1:100; Millipore). Fluorescent secondary antibodies conjugated to either Alexa 488 or 594 (1:200; Invitrogen) were used for detection of primary antibodies.

\section{Analysis of immunohistochemistry: interneuron densities and distribution}

All immunohistochemical images were captured using a confocal microscope (Zeiss LSM510 Meta) at 10× magnification, converted to grey-scale and normalized to background staining. Sections chosen for analysis were anatomically-matched between comparing groups and included samples from rostral, medial and caudal regions. A two-dimensional cell counting approach was employed, with random sampling from fixed regions of interest (ROI) to provide accurate estimates of cell densities [53]. Fluorescent cells within each ROI were counted using the ITCN plugin for ImageJ (http://rsb.info.nih.gov/ij/) (ITCN parameters: width, 20-25 pixels; minimum distance, $10-13$ pixels; threshold, 0.3 pixels) [42,54]. Anatomical regions were defined according to the Golgi Atlas of the Postnatal Mouse Brain [55]. Specific procedures for defining areas of analysis are described below.

\section{Interneuron density}

A fixed rectangular ROI was positioned over the $\mathrm{mPFC}$ ( $1 \mathrm{~mm}$ high $\times 500 \mu \mathrm{m}$ wide) and the DLFC $(750 \mu \mathrm{m}$ high $\times 1.6 \mathrm{~mm}$ ) (Figure 2A). Similarly for the hippocampus, fixed areas were placed on the dentate gyrus (DG) $(300 \mu \mathrm{m} \times 600 \mu \mathrm{m}), \mathrm{CA} 1$ and CA2/3 $(400 \mu \mathrm{m} \times 400 \mu \mathrm{m})$ subfields of the hippocampus for analysis of interneuron subtype densities (Figure 6A). Only PV-labeled cells were counted in the DG as CB-interneurons were absent. Since $\mathrm{CB}$ also labels pyramidal neurons within the hippocampus, CB-labeled interneurons were distinguished 

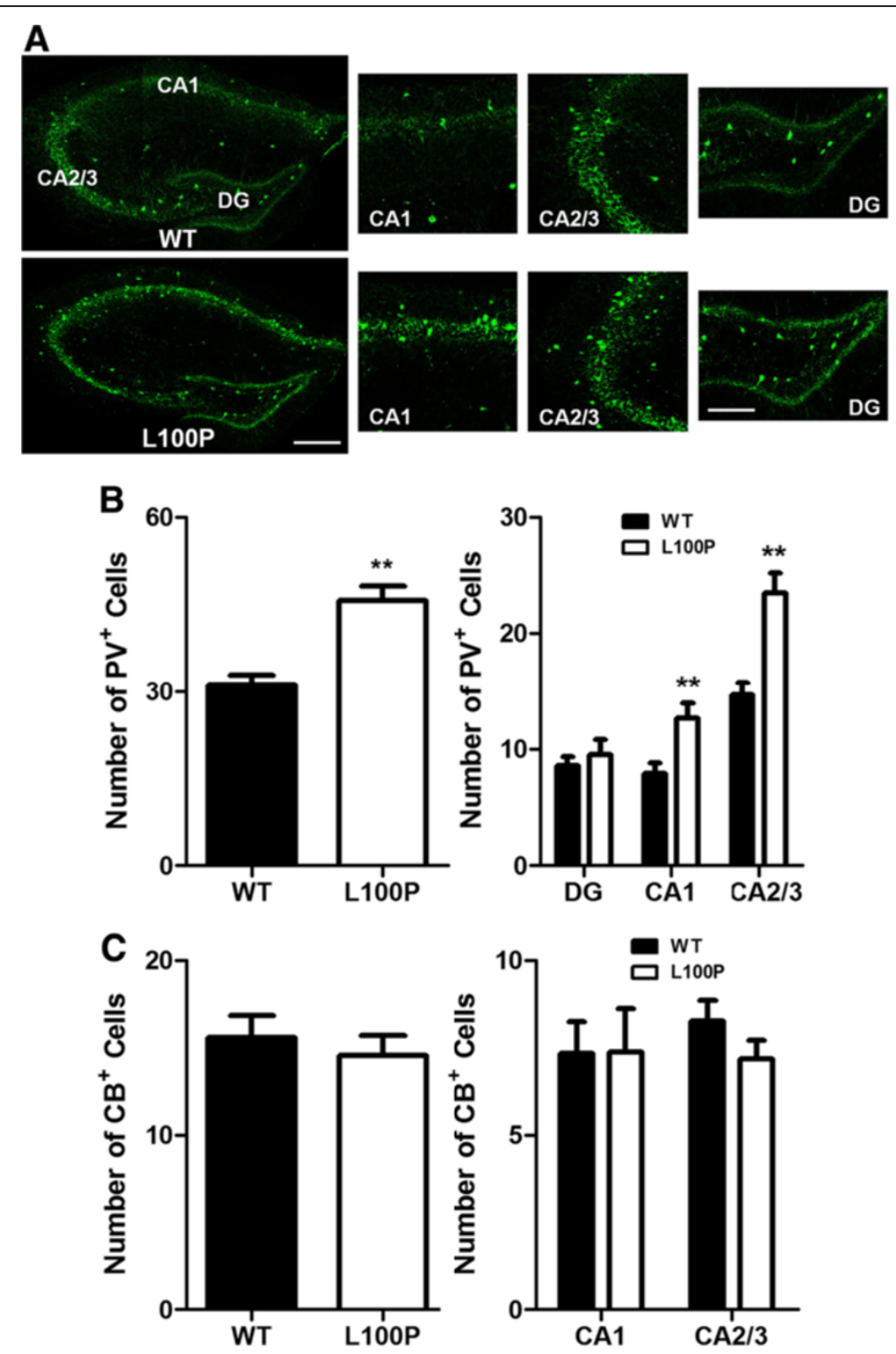

Figure 6 Selective increase of PV-interneurons in CA1 and CA2/3 regions of the hippocampus in Disc1-L100P adult mutant mice.

(A) PV-immunostained images of the hippocampus in WT and Disc1-L100P mice were divided into CA1, CA2/3 and DG subfields in which labeled cells were counted. Scale Bar, $300 \mu \mathrm{m}$. A higher magnification of each subfield is shown on the right. Scale Bar, $100 \mu \mathrm{m}$. (B) Disc1-L100P mutants had significantly more PV-interneurons in the hippocampus, particularly within CA1 and CA2/3 subfields when compared to WT ( $n=12$ ROIs for each region from 4 different mice per group). (C) There was no significant difference in the number of $\mathrm{CB}^{+}$interneurons within the $C A 1$ and CA2/3 regions between WT and Disc1-L100P mutants. DG was excluded from analysis due to the absence of CB-interneurons. All data are shown as mean \pm SEM; ** $p<0.01$ versus WT. CB, calbindin; PV, parvalbumin; CA, Cornu Ammonis; DG, dentate gyrus.

and identified on the basis of their location outside the stratum pyramidale cell layer [56,57].

\section{Interneuron distribution}

In the embryonic E14 and E16 brains, a selected curved region $(300 \mu \mathrm{m}$ wide) from the dorsal cortex to ventral preoptic area was outlined, straightened and divided into seven equal ROIs to capture the tangential migratory paths of newborn interneurons (ImageJ) (Figure 1A).
Analysis of both laminar and tangential interneuron distribution was performed only in adult brains. Four rectangular ROIs (laminar: fixed width of $800 \mu \mathrm{m}$ but variable length spanning the thickness of the cortex; tangential: $1 \mathrm{~mm}$ high $\times 800 \mu \mathrm{m}$ wide) were delineated across the neocortex with the long axis perpendicular to the pial surface (Figure 4A). Specifically for laminar distribution analyses, each ROI was further sub-divided into eight equal regions from the pia mater to the bottom edge of 
layer VI (Figure 3A). For all distribution measurements, the number of fluorescently-labeled cells in each bin was counted and expressed as a percentage of the total number within all bins. A percentage rather than absolute counts was used since the exact area covered by the ROI may differ for each brain section, and our objective was to ascertain a shift in distribution across the ROI.

\section{GAD67 and PV colocalization}

Fluorescent images for GAD67/PV analysis were taken at $25 \times$ magnification. Three fixed square ROIs $(350 \times$ $350 \mu \mathrm{m}^{2}$ ) were positioned over each of the two hemispheres across the neocortex. All images were blinded prior to analysis. Co-localization was defined by the experimenter as any overlap in staining. Both the number of GAD67 ${ }^{+} \mathrm{PV}^{+}$and total $\mathrm{PV}^{+}$cells were counted manually and expressed as a percentage.

\section{Statistical analysis}

Statistical differences between WT and Disc1-L100P mutants were analyzed using the Student's two-tailed $t$-test or two-way ANOVA (SPSS 13.0), followed by Bonferroni's correction for multiple testing. Data are expressed as mean \pm standard error of mean (SEM). A significance level of $p<0.05$ was used for all analyses.

\section{Additional file}

Additional file 1: Figure S1. A decrease in average total number of PV- and CB-interneurons within the MPFC in Disc1-L100P adult mutants. Disc1-L100P mutants had significantly fewer average total PV- and CB-immunostained cells within the MPFC (left) but no significant difference within the DLFC (right) when compared to $W T$ ( $n=16$ from 4 mice per group; $t$-test, $p<0.05$ ). All data are shown as mean $\pm \mathrm{SEM}$; ${ }^{*} p<0.05$ versus WT. CB, calbindin; DLFC, dorsal lateral frontal cortex; mPFC, medial prefrontal cortex; PV, parvalbumin

\section{Abbreviations}

CB: Calbindin; DISC1: Disrupted-in-Schizophrenia-1; DLFC: Dorsolateral frontal cortex; GAD67: Glutamic acid decarboxylase 67; mPFC: Medial prefrontal cortex; PV: Parvalbumin.

\section{Competing interests}

All authors declare that they have no competing financial interests.

\section{Authors' contributions \\ Albert Wong, Sabine Cordes, John Roder and Frankie Lee designed the study. Frankie Lee and Clement Zai performed all experiments and statistical analysis. Frankie Lee and Albert Wong prepared the first draft of the manuscript. All authors contributed to and have approved the final manuscript.}

\section{Acknowledgements}

This work was supported by the Canadian Institutes for Health Research (CIHR), which provided operating funds through a Genomic Medicine and Human Development operating grant GMH79044 and salary support in the form of a Clinician-Scientist Fellowship to AHCW, who also holds a NARSAD Independent Investigator Award and an OMHF Mid-career Investigator Fellowship. CCZ was funded by fellowships from the American Foundation for Suicide Prevention, Eli Lilly, CAMH Foundation. We also thank Carlos Law for blinding of data during analysis. Centre for Addiction and Mental Health, 250 College Street, Room 711, Toronto, Ontario, Canada. M5T 1R8.

\section{Author details}

${ }^{1}$ Campbell Family Mental Health Research Institute, Centre for Addiction and Mental Health, 250 College Street, Toronto, ON M5T 1R8, Canada.

${ }^{2}$ Department of Pharmacology, University of Toronto, Toronto, ON M5S 1A8, Canada. ${ }^{3}$ Department of Molecular Genetics, University of Toronto, Toronto, ON M5S 1A8, Canada. ${ }^{4}$ Samuel Lunenfeld Research Institute, Mount Sinai Hospital, Toronto, ON M5G 1X5, Canada. ${ }^{5}$ Department of Psychiatry, University of Toronto, Toronto, ON M5T 1R8, Canada. ${ }^{6}$ Institute of Medical Sciences, University of Toronto, Toronto, ON M5S 1A8, Canada.

Received: 19 March 2013 Accepted: 27 April 2013

Published: 30 April 2013

\section{References}

1. Miller EK, Cohen JD: An integrative theory of prefrontal cortex function. Annu Rev Neurosci 2001, 24:167-202.

2. Marin O: Interneuron dysfunction in psychiatric disorders. Nat Rev Neurosci 2012, 13:107-120.

3. Gonzalez-Burgos G, Hashimoto T, Lewis DA: Alterations of cortical GABA neurons and network oscillations in schizophrenia. Curr Psychiatry Rep 2010, 12:335-344.

4. Blum BP, Mann Jj: The GABAergic system in schizophrenia. Int J Neuropsychopharmacol 2002, 5:159-179.

5. Lewis DA, Hashimoto T, Volk DW: Cortical inhibitory neurons and schizophrenia. Nat Rev Neurosci 2005, 6:312-324.

6. Markram H, Toledo-Rodriguez M, Wang Y, Gupta A, Silberberg G, Wu C: Interneurons of the neocortical inhibitory system. Nat Rev Neurosci 2004, 5:793-807.

7. Akbarian S, Kim JJ, Potkin SG, Hagman JO, Tafazzoli A, Bunney WE Jr, Jones EG: Gene expression for glutamic acid decarboxylase is reduced without loss of neurons in prefrontal cortex of schizophrenics. Arch Gen Psychiatry 1995, 52:258-266.

8. Hashimoto T, Volk DW, Eggan SM, Mirnics K, Pierri JN, Sun Z, Sampson AR, Lewis DA: Gene expression deficits in a subclass of GABA neurons in the prefrontal cortex of subjects with schizophrenia. J Neurosci 2003, 23:6315-6326.

9. Cardin JA, Carlen M, Meletis K, Knoblich U, Zhang F, Deisseroth K, Tsai LH, Moore Cl: Driving fast-spiking cells induces gamma rhythm and controls sensory responses. Nature 2009, 459:663-667.

10. Sohal VS, Zhang F, Yizhar O, Deisseroth K: Parvalbumin neurons and gamma rhythms enhance cortical circuit performance. Nature 2009, 459:698-702.

11. Haenschel C, Bittner RA, Waltz J, Haertling F, Wibral M, Singer W, Linden DE, Rodriguez E: Cortical oscillatory activity is critical for working memory as revealed by deficits in early-onset schizophrenia. J Neurosci 2009, 29:9481-9489

12. Howard MW, Rizzuto DS, Caplan JB, Madsen JR, Lisman J, AschenbrennerScheibe R, Schulze-Bonhage A, Kahana MJ: Gamma oscillations correlate with working memory load in humans. Cereb Cortex 2003, 13:1369-1374.

13. Minzenberg MJ, Firl AJ, Yoon JH, Gomes GC, Reinking C, Carter CS: Gamma oscillatory power is impaired during cognitive control independent of medication status in first-episode schizophrenia. Neuropsychopharmacology 2010, 35:2590-2599.

14. Uhlhaas PJ, Singer W: Abnormal neural oscillations and synchrony in schizophrenia. Nat Rev Neurosci 2010, 11:100-113.

15. Lodge DJ, Behrens MM, Grace AA: A loss of parvalbumin-containing interneurons is associated with diminished oscillatory activity in an animal model of schizophrenia. J Neurosci 2009, 29:2344-2354.

16. Carlen M, Meletis K, Siegle JH, Cardin JA, Futai K, Vierling-Claassen D, Ruhlmann C, Jones SR, Deisseroth $K$, Sheng M, et al: A critical role for NMDA receptors in parvalbumin interneurons for gamma rhythm induction and behavior. Mol Psychiatry 2012, 17:537-548.

17. Millar JK, Wilson-Annan JC, Anderson S, Christie S, Taylor MS, Semple CA Devon RS, Clair DM, Muir WJ, Blackwood DH, Porteous DJ: Disruption of two novel genes by a translocation co-segregating with schizophrenia. Hum Mol Genet 2000, 9:1415-1423.

18. Chubb JE, Bradshaw NJ, Soares DC, Porteous DJ, Millar JK: The DISC locus in psychiatric illness. Mol Psychiatry 2008, 13:36-64. 
19. Bradshaw NJ, Porteous DJ: DISC1-binding proteins in neural development, signalling and schizophrenia. Neuropharmacology 2012, 62:1230-1241.

20. Brandon NJ, Sawa A: Linking neurodevelopmental and synaptic theories of mental illness through DISC1. Nat Rev Neurosci 2011, 12:707-722.

21. Hikida T, Jaaro-Peled H, Seshadri S, Oishi K, Hookway C, Kong S, Wu D, Xue $\mathrm{R}$, Andrade M, Tankou S, et al: Dominant-negative DISC1 transgenic mice display schizophrenia-associated phenotypes detected by measures translatable to humans. Proc Natl Acad Sci U S A 2007, 104:14501-14506.

22. Shen S, Lang B, Nakamoto C, Zhang F, Pu J, Kuan SL, Chatzi C, He S, Mackie I, Brandon NJ, et al: Schizophrenia-related neural and behavioral phenotypes in transgenic mice expressing truncated Disc1. J Neurosci 2008, 28:10893-10904.

23. Ayhan Y, Abazyan B, Nomura J, Kim R, Ladenheim B, Krasnova IN, Sawa A, Margolis RL, Cadet JL, Mori S, et al: Differential effects of prenatal and postnatal expressions of mutant human DISC1 on neurobehavioral phenotypes in transgenic mice: evidence for neurodevelopmental origin of major psychiatric disorders. Mol Psychiatry 2011, 16:293-306.

24. Niwa M, Kamiya A, Murai R, Kubo K, Gruber AJ, Tomita K, Lu L, Tomisato S, Jaaro-Peled H, Seshadri S, et al: Knockdown of DISC1 by in utero gene transfer disturbs postnatal dopaminergic maturation in the frontal cortex and leads to adult behavioral deficits. Neuron 2010, 65:480-489.

25. Steinecke A, Gampe C, Valkova C, Kaether C, Bolz J: Disrupted-inSchizophrenia 1 (DISC1) is necessary for the correct migration of cortical interneurons. J Neurosci 2012, 32:738-745.

26. Clapcote SJ, Lipina TV, Millar JK, Mackie S, Christie S, Ogawa F, Lerch JP, Trimble K, Uchiyama M, Sakuraba Y, et al: Behavioral phenotypes of Disc1 missense mutations in mice. Neuron 2007, 54:387-402.

27. Marx J: Behavioral genetics. Evidence linking DISC1 gene to mental illness builds. Science 2007, 318:1062-1063.

28. Jaaro-Peled H, Hayashi-Takagi A, Seshadri S, Kamiya A, Brandon NJ, Sawa A Neurodevelopmental mechanisms of schizophrenia: understanding disturbed postnatal brain maturation through neuregulin-1-ErbB4 and DISC1. Trends Neurosci 2009, 32:485-495.

29. Anderson SA, Eisenstat DD, Shi L, Rubenstein JL: Interneuron migration from basal forebrain to neocortex: dependence on Dlx genes. Science 1997, 278:474-476.

30. Beasley CL, Reynolds GP: Parvalbumin-immunoreactive neurons are reduced in the prefrontal cortex of schizophrenics. Schizophr Res 1997, 24:349-355.

31. Nakajima K: Control of tangential/non-radial migration of neurons in the developing cerebral cortex. Neurochem Int 2007, 51:121-131.

32. Heckers S, Konradi C: Hippocampal pathology in schizophrenia. Curr Top Behav Neurosci 2010, 4:529-553.

33. Yau HJ, Wang HF, Lai C, Liu FC: Neural development of the neuregulin receptor ErbB4 in the cerebral cortex and the hippocampus: preferential expression by interneurons tangentially migrating from the ganglionic eminences. Cereb Cortex 2003, 13:252-264.

34. Li H, Chou SJ, Hamasaki T, Perez-Garcia CG, O'Leary DD: Neuregulin repellent signaling via ErbB4 restricts GABAergic interneurons to migratory paths from ganglionic eminence to cortical destinations. Neural Dev 2012, 7:10.

35. Flames N, Long JE, Garratt AN, Fischer TM, Gassmann M, Birchmeier C, Lai C, Rubenstein JL, Marin O: Short- and long-range attraction of cortical GABAergic interneurons by neuregulin-1. Neuron 2004, 44:251-261.

36. Polleux F, Whitford KL, Dijkhuizen PA, Vitalis T, Ghosh A: Control of cortical interneuron migration by neurotrophins and Pl3-kinase signaling. Development 2002, 129:3147-3160.

37. Batista-Brito R, Rossignol E, Hjerling-Leffler J, Denaxa M, Wegner M, Lefebvre V, Pachnis V, Fishell G: The cell-intrinsic requirement of Sox6 for cortical interneuron development. Neuron 2009, 63:466-481.

38. Azim E, Jabaudon D, Fame RM, Macklis JD: SOX6 controls dorsal progenitor identity and interneuron diversity during neocortical development. Nat Neurosci 2009, 12:1238-1247.

39. Cobos I, Borello U, Rubenstein JL: Dlx transcription factors promote migration through repression of axon and dendrite growth. Neuron 2007, 54:873-888.

40. Wonders CP, Anderson SA: The origin and specification of cortical interneurons. Nat Rev Neurosci 2006, 7:687-696.

41. Gonzalez-Burgos G, Fish KN, Lewis DA: GABA neuron alterations, cortical circuit dysfunction and cognitive deficits in schizophrenia. Neural Plast 2011, 2011:723184.
42. Lee FH, Fadel MP, Preston-Maher K, Cordes SP, Clapcote SJ, Price DJ, Roder JC, Wong AH: Disc1 Point Mutations in Mice Affect Development of the Cerebral Cortex. J Neurosci 2011, 31:3197-3206.

43. Lewis DA, Curley AA, Glausier JR, Volk DW: Cortical parvalbumin interneurons and cognitive dysfunction in schizophrenia. Trends Neurosci 2012, 35:57-67.

44. Benes FM, Kwok EW, Vincent SL, Todtenkopf MS: A reduction of nonpyramidal cells in sector CA2 of schizophrenics and manic depressives. Biol Psychiatry 1998, 44:88-97.

45. Zhang ZJ, Reynolds GP: A selective decrease in the relative density of parvalbumin-immunoreactive neurons in the hippocampus in schizophrenia. Schizophr Res 2002, 55:1-10.

46. Torrey EF, Barci BM, Webster MJ, Bartko JJ, Meador-Woodruff JH, Knable MB: Neurochemical markers for schizophrenia, bipolar disorder, and major depression in postmortem brains. Biol Psychiatry 2005, 57:252-260.

47. Daviss SR, Lewis DA: Local circuit neurons of the prefrontal cortex in schizophrenia: selective increase in the density of calbindin-immunoreactive neurons. Psychiatry Res 1995, 59:81-96.

48. Fung SJ, Webster MJ, Sivagnanasundaram S, Duncan C, Elashoff M, Weickert CS: Expression of interneuron markers in the dorsolateral prefrontal cortex of the developing human and in schizophrenia. Am J Psychiatry 2010, 167:1479-1488.

49. Kamiya A, Kubo K, Tomoda T, Takaki M, Youn R, Ozeki Y, Sawamura N, Park U, Kudo C, Okawa M, et al: A schizophrenia-associated mutation of DISC1 perturbs cerebral cortex development. Nat Cell Biol 2005, 7:1167-1178.

50. Duan X, Chang JH, Ge S, Faulkner RL, Kim JY, Kitabatake Y, Liu XB, Yang CH, Jordan JD, Ma DK, et al: Disrupted-In-Schizophrenia 1 regulates integration of newly generated neurons in the adult brain. Cell 2007, 130:1146-1158.

51. Meyer KD, Morris JA: Immunohistochemical analysis of Disc1 expression in the developing and adult hippocampus. Gene Expr Patterns 2008, 8:494-501.

52. Mesbah-Oskui L: Place cell activity in Disc1-L100P mutant mice. Toronto: University of Toronto, Department of Physiology; 2011.

53. Benes FM, Lange N: Two-dimensional versus three-dimensional cell counting: a practical perspective. Trends Neurosci 2001, 24:11-17.

54. Usaj M, Torkar D, Kanduser M, Miklavcic D: Cell counting tool parameters optimization approach for electroporation efficiency determination of attached cells in phase contrast images. J Microsc 2011, 241:303-314.

55. Valverde F: Golgi Atlas of the Postnatal Mouse Brain. Austria: Springer Wien New York; 1998.

56. Toth K, Freund TF: Calbindin D28k-containing nonpyramidal cells in the rat hippocampus: their immunoreactivity for GABA and projection to the medial septum. Neuroscience 1992,49:793-805.

57. Danglot L, Triller A, Marty S: The development of hippocampal interneurons in rodents. Hippocampus 2006, 16:1032-1060.

doi:10.1186/1756-6606-6-20

Cite this article as: Lee et al:: Abnormal interneuron development in disrupted-in-schizophrenia-1 L100P mutant mice. Molecular Brain 2013 6:20.

\section{Submit your next manuscript to BioMed Central and take full advantage of:}

- Convenient online submission

- Thorough peer review

- No space constraints or color figure charges

- Immediate publication on acceptance

- Inclusion in PubMed, CAS, Scopus and Google Scholar

- Research which is freely available for redistribution 